\title{
A Validated Enantioselective Assay for the Determination of Ibuprofen in Human Plasma Using Ultra Performance Liquid Chromatography with Tandem Mass Spectrometry (UPLC-MS/MS)
}

\author{
András Szeitz ${ }^{1 *}$, Andrea Nicole Edginton ${ }^{2}$, Henry Tao Peng ${ }^{3}$, Bob Cheung ${ }^{3}$, Kenneth Wayne Riggs ${ }^{1}$ \\ ${ }^{1}$ Faculty of Pharmaceutical Sciences, The University of British Columbia, Vancouver, Canada \\ ${ }^{2}$ School of Pharmacy, University of Waterloo, Waterloo, Canada \\ ${ }^{3}$ Defence Research and Development Canada-Toronto, Toronto, Canada \\ E-mail: szeitz@interchange.ubc.ca \\ Received April 8, 2010; revised July 13, 2010; accepted July 20, 2010
}

\begin{abstract}
A modified ultra performance liquid chromatography-tandem mass spectrometry (UPLC-MS/MS) method was developed and validated for the quantitation of ibuprofen enantiomers in human plasma. Ibuprofen and flurbiprofen (internal standard) were extracted from human plasma at acidic $\mathrm{pH}$, using a single-step liquid-liquid extraction with methyl-tert-butyl ether. The enantiomers of ibuprofen and flurbiprofen were derivatized to yield the corresponding diastereomers. Chromatographic separation was achieved using a phenyl column with a run time of $20 \mathrm{~min} .(R)$ - and $(S)$-ibuprofen were quantitated at the multiple reaction monitoring (MRM) transition of $\mathrm{m} / \mathrm{z} 360.2 \rightarrow 232.1$, and $(R)$ - and $(S)$-flurbiprofen were monitored at the MRM transition of $m / z 398.3 \rightarrow 270.1$. The method was validated for accuracy, precision, linearity, range, limit of quantitation (LOQ), limit of detection (LOD), selectivity, absolute recovery, matrix effect, dilution integrity, and evaluation of carry-over. Accuracy for $(R)$-ibuprofen ranged between $-11.8 \%$ and $11.2 \%$, and for $(S)$-ibuprofen between $-8.6 \%$ and $-0.3 \%$. Precision for $(R)$-ibuprofen was $\leq 11.2 \%$, and for $(S)$-ibuprofen $\leq$ $7.0 \%$. The calibration curves were weighted $\left(1 / \mathrm{X}^{2}, \mathrm{n}=7\right)$ and were linear with $\mathrm{r}^{2}$ for $(R)$-ibuprofen $\geq 0.988$ and for $(S)$-ibuprofen $\geq 0.990$. The range of the method was 50 to $5000 \mathrm{ng} / \mathrm{mL}$ with the LOQ of $50 \mathrm{ng} / \mathrm{mL}$, and LOD of $1 \mathrm{ng} / \mathrm{mL}$, for $(R)$ - and $(S)$-ibuprofen requiring $100 \mu \mathrm{L}$ of sample. The method was applied successfully to a pharmacokinetic study with the administration of a single oral dose of ibuprofen capsules to human subjects.
\end{abstract}

Keywords: Ibuprofen Enantiomers, UPLC-MS/MS, Human Plasma, Method Validation, Pharmacokinetics

\section{Introduction}

Ibuprofen, a member of the 2-substituted arylpropionic acid (2-APA) family, is a non-steroidal anti-inflammatory drug (NSAID), which is used to treat moderate pain, fever, rheumatic disorders and related inflammatory diseases. Ibuprofen, one of the most popular "profen" drugs, was first marketed in the UK in 1969, and has been commercialized as a racemate drug. In Switzerland and Austria, in addition to the racemate drug, the $(S)$-enantiomer is also sold.

Ibuprofen undergoes chiral inversion in vitro [1] and in vivo, during metabolism in rats, mouse, rabbits [2-4] and humans $[5,6]$ and shows stereoselective pharmacological effects $[7,8]$ metabolism, pharmacokinetics [9-11] and disposition $[12,13]$. It has been reported also that ibuprofen is extensively bound to proteins in plasma in humans $[11,14,15]$.

Several methodologies are available for the determination of ibuprofen. These techniques include direct or indirect high performance liquid chromatography (HPLC) methods. In the direct HPLC methods, a chiral stationary phase is used and the enantiomers are analyzed without sample derivatization. Certain direct HPLC methods employ ultra-violet (UV) detection using $\alpha_{1}$-acid glycoprotein [16-19], $\beta$-cyclodextrin [20], cellulose [21], polysaccharide [22], and amylose derivative [23] chiral sta- 
tionary phases (CSPs). A cellulose derivative CSP using radiometric and tandem mass spectrometric (MS/MS) detection [24] and an amylose derivative CSP using MS/MS [25] were also employed for the determination of ibuprofen enantiomers.

The direct HPLC methods have the advantage that the ibuprofen enantiomers can be analyzed without derivatization, however, these assays have poor sensitivity, reproducibility, require a large sample volume [16,18-20] or lack the satisfactory chromatographic baseline separation of the stereoisomer peaks [20,25]. Some direct HPLC methods achieved good sensitivity and use a small sample volume, but due to the non-selective characteristics of the UV detection they require extended analysis time to avoid interferences between the ibuprofen enantiomers and the co-eluting endogenous components from the biological matrix [23]. Further direct HPLC assays employ radiometric and selective mass spectrometric detection, but they are reported for in vitro applications, and not for the quantitation of the ibuprofen enantiomers over a concentration range in body fluids [24].

In the indirect HPLC methods, a reverse-phase stationary phase is used and the enantiomers are analyzed after sample derivatization to yield their corresponding diastereomers. The indirect methods have improved sensitivity and are better suited for the analysis of ibuprofen enantiomers in a variety of complex biological matrices. The indirect techniques include HPLC using UV [26,27], fluorescence $[28,29]$, and mass spectrometric detection [30-33]. Gas chromatography-mass spectrometry ( $\mathrm{GC} /$ MS) methods have been reported as well [2,6,34-36].

While the indirect HPLC methods using UV and fluorescence detection are suitable for the enantioselective determination of $(R)$ - and $(S)$-ibuprofen in biological samples, they still lack the satisfactory sensitivity (e.g., limit of quantitation, $\mathrm{LOQ} \geq 0.1 \mu \mathrm{g} / \mathrm{mL}$ ) and use a large sample volume (i.e., $0.5 \mathrm{~mL}$ plasma or serum) [26,27, 29], therefore, they may not be used satisfactorily for detailed pharmacokinetic studies over prolonged time periods. GC/MS methods can be stereoselective, but they use a larger sample size for analysis (i.e., $0.8 \mathrm{~mL}$ plasma or $1 \mathrm{~mL}$ of serum) $[34,6]$, and their sensitivity is insufficient (i.e., $0.25 \mu \mathrm{g} / \mathrm{mL}$ or LOQ $5 \mu \mathrm{g} / \mathrm{mL}$ ) [34,35], or they are not stereoselective $[35,36]$. Several indirect HPLC/ MS/MS methods were reported for various in vitro [30-32] and in vivo [33] analyses of ibuprofen, but these methods are not stereoselective and do not represent significant improvement in comparison to the methodologies reported above.

In the present study, an enantioselective ultra performance liquid chromatography-tandem mass spectrometry (UPLC-MS/MS) method with improved sensitivity is presented for the quantitation of $(R)$ - and $(S)$-ibuprofen enantiomers in human plasma using $(R)$ and $(S)$-flurbiprofen as internal standards. The assay was validated and applied successfully to the pharmacoki- netic study of orally administered ibuprofen capsules to humans.

\section{Experimental}

\subsection{Chemicals and Standards}

(R)-(-)-ibuprofen (purity 98\%), and (S)-(+)-ibuprofen (purity 98\%), were purchased from Toronto Research Chemicals Inc. (North York, ON, Canada). (S)-(+)-Flurbiprofen (purity 98\%), (R)-(-)-Flurbiprofen (purity 97\%), internal standards, N-(3-Dimethylaminopropyl)-N'-ethyl-carbodiimide hydrochloride (CDI) (purity $>98 \%),(\mathrm{R})-(+)-1-(1-$ Naphtyl)-ethylamine ((R)-NEA) (purity > 99\%) were purchased from Sigma-Aldrich (St. Louis, MO, USA), and 1-hydroxybenzotriazole (HOBt:H2O) was purchased from AnaSpec, Inc. (San Jose, CA, USA). Methyl-tertbutyl ether, acetonitrile, methanol (HPLC grade) were purchased from Fisher Scientific (Fair Lawn, NY, USA), and dichloromethane (HPLC Grade) was obtained from Acros Organics (Geel, Belgium). Ammonium Acetate (AnalaR grade) was obtained from BDH Inc., (Toronto, ON, Canada), formic acid (puriss. p.a. for mass spectroscopy) was purchased from Fluka (Steinheim, Germany), hydrochloric acid $(1.0 \mathrm{~N})$ was purchased from VWR (West Chester, PA, USA). Blank human plasma (Sodium EDTA-treated) was purchased from Bioreclamation, Inc. (Westbury NY, USA). Ultra pure water was prepared in our laboratory using a Milli-Q Synthesis system (Millipore, Billerica, MA, USA). Ibuprofen capsules (Advil $^{\mathrm{TM}}$ ibuprofen $400 \mathrm{mg}$ Liquid Filled Capsules, Extra Strength Liqui-Gels, Wyeth Consumer Healthcare Inc., Mississauga, ON, Canada) were obtained from Shoppers Drug Mart, Toronto, ON, Canada.

\subsection{Instrumentation and Experimental Conditions}

The UPLC-MS/MS system consisted of a Waters Acquity UPLC Binary Solvent Manager and a Waters Acquity UPLC Sample Manager connected to a Waters Quattro Premier XE triple quadrupole mass spectrometer. The mass spectrometer was operated in electrospray positive ionization $(\mathrm{ES}+)$ mode, and data were acquired using a MassLynx v. 4.1 software on a Microsoft Windows XP Professional operating platform.

Chromatographic separation was achieved using a Waters Acquity UPLC BEH Phenyl $1.7 \mu \mathrm{m}, 2.1 \times 150$ $\mathrm{mm}$ column maintained at $30^{\circ} \mathrm{C}$, and the autosampler tray temperature was maintained at $10^{\circ} \mathrm{C}$. Solvent a was water containing $10 \mathrm{mM}$ ammonium acetate and $0.1 \%$ formic acid, and solvent $\mathrm{b}$ was a mixture of $64 \%$ acetonitrile $36 \%$ methanol containing $10 \mathrm{mM}$ ammonium acetate and $0.1 \%$ formic acid. The mobile phase initial conditions were solvent a (35\%) and solvent b (65\%) 
with a flow rate of $0.2 \mathrm{~mL} / \mathrm{min}$, which were maintained for $12 \mathrm{~min}(0-12 \mathrm{~min})$. At $12.1 \mathrm{~min}$, solvent $\mathrm{b}$ was increased to $100 \%$ and the flow rate was gradually increased to $0.5 \mathrm{~mL} / \mathrm{min}$ by $14 \mathrm{~min}$ (12.1-14 min) and held for $1 \mathrm{~min}$ (14-15 $\mathrm{min})$. At $15.1 \mathrm{~min}$, the initial conditions were set and the column was equilibrated for $4.9 \mathrm{~min}$ (15.1-20 min). The total run time was $20 \mathrm{~min}$, the injection volume was $15 \mu \mathrm{L}$.

Mass spectrometric conditions were as follows: capillary voltage $3 \mathrm{kV}$, cone voltage $30 \mathrm{~V}$, source temperature $120^{\circ} \mathrm{C}$, desolvation gas temperature $300^{\circ} \mathrm{C}$, desolvation gas flow $1000 \mathrm{~L} /$ hour. Diastereomeric amide derivatives of $(R)$ - and $(S)$-ibuprofen were quantitated in multiple reaction monitoring (MRM) using the transition of $\mathrm{m} / \mathrm{z}$ $360.2 \rightarrow 232.1$ at collision energy (CE) $10 \mathrm{eV}$. The dwell time was set to $25 \mathrm{~ms}$. Diastereomeric amide derivatives of $(R)$ - and $(S)$-flurbiprofen were monitored in MRM using the transition of $\mathrm{m} / \mathrm{z} 398.3 \rightarrow 270.1$ at CE $10 \mathrm{eV}$. The dwell time was set to $50 \mathrm{~ms}$. To protect the mass spectrometer from contamination from the samples and to reduce the solvent load in the source, the mobile phase flow was diverted to the waste before $7 \mathrm{~min}$ and after 12 min during the chromatographic run.

\subsection{Preparation of Stock Solutions and Calibration Standards}

Two separate master stock solutions of $(R)$-ibuprofen $(100 \mu \mathrm{g} / \mathrm{mL})$, and $(S)$-ibuprofen $(100 \mu \mathrm{g} / \mathrm{mL})$ enantiomers were prepared in methanol. The $(R)$ - and $(S)$-ibuprofen master stock solutions were combined in equal parts into a mixed working stock solution of $(R)$ and (S)-ibuprofen $(50 \mu \mathrm{g} / \mathrm{mL}$, each). The mixed working stock solution was further diluted with water to yield a series of diluted working stock solutions. The series of diluted working stock solutions were used to prepare the calibration standards in human plasma. The calibration standards were prepared by spiking $10 \mu \mathrm{L}$ aliquots of appropriately diluted working stock solutions into $90 \mu \mathrm{L}$ aliquots of blank human plasma yielding the final volume of $100 \mu \mathrm{L}$ calibration standards in human plasma. Calibration standards were prepared freshly on the day of a batch analysis in the concentration range of $50 \mathrm{ng} / \mathrm{mL}$ to $5000 \mathrm{ng} / \mathrm{mL}$ for $(R)$ - and $(S)$-ibuprofen. The solutions were stored at $4{ }^{\circ} \mathrm{C}$ until analysis.

The internal standard solutions were prepared as follows. Two separate master stock solutions of $(R)$-flurbiprofen $(100 \mu \mathrm{g} / \mathrm{mL})$, and $(S)$-flurbiprofen $(100 \mu \mathrm{g} / \mathrm{mL})$ enantiomers were prepared in methanol. The $(R)$ - and $(S)$-flurbiprofen master stock solutions were combined in equal parts and diluted with water into a mixed working stock solution of $(R)$ - and $(S)$-flurbiprofen $(10 \mu \mathrm{g} / \mathrm{mL}$, each). The mixed working stock solution was further diluted with water to yield the diluted working stock solution of $(R)$ - and $(S)$-flurbiprofen (100 $\mathrm{ng} / \mathrm{mL}$, each). The solutions were stored at $4^{\circ} \mathrm{C}$ until analysis.

\subsection{Preparation of Quality Control Samples}

Quality control (QC) samples were prepared as QC-Low (80 ng/mL), QC-Mid (250 ng/mL), and QC-High (2500 $\mathrm{ng} / \mathrm{mL}$ ) samples in human plasma. A volume of $5 \mathrm{~mL}$ of QC samples at each concentration level was prepared. The QC-High samples were prepared by spiking appropriate volumes of each of the master stock solutions of $(R)$-ibuprofen $(100 \mu \mathrm{g} / \mathrm{mL})$, and $(S)$-ibuprofen $(100 \mu \mathrm{g} / \mathrm{mL})$ enantiomers in methanol into blank human plasma, and the QC-Mid, and QC-Low samples were prepared by spiking the appropriately diluted mixed working stock solutions of $(R)$ - and $(S)$-ibuprofen into blank human plasma. The QC samples were dispensed in equal aliquots (approx. $130 \mu \mathrm{L}$ ) into vials and stored at $-80^{\circ} \mathrm{C}$ until use. For each batch analysis, fresh aliquots of QC-Low, QC-Mid, and QC-High samples were thawed, analyzed, and then discarded.

\subsection{Preparation of Reagent Solutions}

Stock solutions of CDI and $(R)$-NEA $(1 \mathrm{mg} / \mathrm{mL})$ were prepared in dichloromethane. Stock solution of $\mathrm{HOBt}$ Hydrate $(1 \mathrm{mg} / \mathrm{mL})$ was prepared in dichloromethane containing $10 \%$ acetonitrile. The stock solutions were stored at $4^{\circ} \mathrm{C}$ until use.

\subsection{Sample Preparation}

Human plasma samples were stored at $-80^{\circ} \mathrm{C}$ until analysis. On the day of sample analysis, the samples were thawed at room temperature and processed with the method. All human plasma samples, with the exception of the 0-min time point samples, were diluted into the range of the calibration curve by mixing $10 \mu \mathrm{L}$ aliquots of human plasma samples with $90 \mu \mathrm{L}$ aliquots of blank human plasma yielding a final volume of $100 \mu \mathrm{L}$ of sample (i.e., 10-fold dilution). In disposable borosilicate glass tubes, to $100 \mu \mathrm{L}$ of samples, aliquots of $100 \mu \mathrm{L}$ of the diluted working stock solution of the internal standards $((R)$ - and $(S)$-flurbiprofen, $100 \mathrm{ng} / \mathrm{mL}$, each) were added followed by the addition of $200 \mu \mathrm{L}$ of $1 \mathrm{~N} \mathrm{HCl}$ solution. The samples were vortex-mixed for at least 15 seconds, and $3.0 \mathrm{~mL}$ of methyl-tert-butyl ether was added to the tubes. The samples were vortex-mixed for at least 45 seconds, and the tubes were placed at $-80^{\circ} \mathrm{C}$ for at least $10 \mathrm{~min}$. The tubes were removed from the $-80^{\circ} \mathrm{C}$ freezer and the top layers were transferred to a new set of tubes. The organic layer was brought to dryness in a sample evaporator, under nitrogen, at $c a .35^{\circ} \mathrm{C}$, and the dried residues were derivatized according to a previously reported procedure [29] with modification. In short, to the dried residues, aliquots of $100 \mu \mathrm{L}$ of CDI solution (1 $\mathrm{mg} / \mathrm{mL}), 100 \mu \mathrm{L}$ of HOBt Hydrate solution $(1 \mathrm{mg} / \mathrm{mL})$, and $100 \mu \mathrm{L}$ of $(R)$-NEA solution $(1 \mathrm{mg} / \mathrm{mL})$ were added, and the mixtures were kept in a dark environment, at 
room temperature, for 2 hours. The samples were then brought to dryness in a sample evaporator, under nitrogen, at $c a .35^{\circ} \mathrm{C}$, and the dried residues were reconstituted with $200 \mu \mathrm{L}$ of acetonitrile/water : 50/50 mixture containing $0.1 \%$ formic acid. The samples were analyzed with UPLC-MS/MS.

\subsection{Method Validation}

The method was validated for accuracy, precision, linearity, range, limit of quantitation (LOQ), limit of detection (LOD), selectivity, absolute recovery, matrix effect, dilution integrity, and evaluation of carry-over in human plasma using $100 \mu \mathrm{L}$ of sample. Calibration curves included six to seven calibration levels and were prepared on each day of batch analysis with a matrix blank. QC-Low, QC-Mid, and QC-High samples were freshly thawed on each day of batch analysis and $100 \mu \mathrm{L}$ aliquots were used during the validation process.

\subsubsection{Accuracy}

Six replicate spiked samples of QC-Low, QC-Mid, and QC-High were prepared in human plasma, and analyzed. Accuracy was expressed as the percentage deviation of the measured $(R)$ - and (S)-ibuprofen concentration against the added concentration, according to the following formula: $\%$ Deviation $=[$ (measured amount/added amount $\times 100]-100$ with negative $\%$ Deviation representing under-estimation, and positive \%Deviation representing over-estimation of the true value. The acceptance criteria for accuracy were $\%$ Deviation $\pm 20 \%$ for the QC-Low samples, and $\pm 15 \%$ for QC-Mid, and QCHigh samples.

For intra-day accuracy, six replicates of QC-Low, QCMid, and QC-High samples were prepared and analyzed on the same day. Intra-day accuracy experiments were repeated for three separate days. For inter-day accuracy, six replicates of QC-Low, QC-Mid, and QC-High were prepared and analyzed on three separate days.

\subsubsection{Precision}

A single spiked sample for QC-Low, QC-Mid, and QC-High (5 mL each) was prepared in human plasma. Six aliquots were removed from each of the QC-Low, QC-Mid, and QC-High samples and analyzed. The relative standard deviation (\%RSD) of the $(R)$ - and $(S)$-ibuprofen concentrations measured in each QC sample were calculated. The acceptance criteria for precision were $\%$ RSD $\leq 20 \%$ for the QC-Low samples, and $\%$ RSD $\leq$ $15 \%$ for the QC-Mid, and QC-High samples.

For intra-day precision, six aliquots were removed from each of the QC-Low, QC-Mid, and QC-High spiked samples and analyzed on the same day. Intra-day precision experiments were repeated for three separate days. For inter-day precision, six aliquots were removed from each of the QC-Low, QC-Mid, and QC-High spiked samples and analyzed on three separate days.

\subsubsection{Linearity and Range}

Calibration curves were prepared for each batch analysis in the following concentrations: 50, 100, 200, 300, 400, $1000,5000 \mathrm{ng} / \mathrm{mL}$ in human plasma. Calibration curves were constructed by plotting the concentrations of $(R)$ and $(S)$-ibuprofen on the X-axis, vs. the chromatographic peak area ratio of $(R)$ - and $(S)$-ibuprofen to $(R)$ - and $(S)$-flurbiprofen internals standards on the Y-axis. Linear regression analyses were performed using the calibration curve data. At least six out of seven of the calibration standards were used to construct the calibration curves. Using the $\mathrm{y}=\mathrm{mx}+\mathrm{b}$ equation, the $\mathrm{y}$-intercept (b), slope (m) and correlation coefficient ( $\mathrm{r}$ ) were calculated. The calibration curves were weighted using the weighting factor of $1 / \mathrm{X}^{2}$. The acceptance criterion for linearity was the coefficient of determination $\mathrm{r}^{2} \geq 0.980$ for $(R)$ - and $(S)$-ibuprofen after weighting with $1 / \mathrm{X}^{2}$. (R)- and $(S)$-ibuprofen concentrations were calculated by the MassLynx software using the following formula: $\mathrm{x}=$ $(\mathrm{y}-\mathrm{b}) / \mathrm{m}$, where $\mathrm{y}=(R)$ - and $(S)$-ibuprofen to IS peak area ratio, $\mathrm{b}=$ weighted $\mathrm{y}$-intercept, $\mathrm{m}=$ weighted slope. The range of the assay was established as the section of the calibration curve where the curve was linear, i.e., $\mathrm{r}^{2} \geq$ 0.980 , the calibration levels were accurate $(\%$ Deviation \pm $15 \%)$ and precise ( $\%$ RSD $\leq 15 \%)$.

\subsubsection{Limit of Quantitation and Limit of Detection}

To determine LOQ, six replicates of the $50 \mathrm{ng} / \mathrm{mL}$ calibration standard were prepared in human plasma and analyzed. The mean response (i.e., signal-to-noise ratio, $\mathrm{S} / \mathrm{N})$, accuracy and precision were determined from the samples. The LOQ was determined as the lowest concentration of the calibration curve which met the following acceptance criteria. The mean response of $(R)$ - and $(S)$ - ibuprofen peaks in the samples was at least 5-times the response compared to the blank sample (the response, $\mathrm{S} / \mathrm{N}$, was calculated using MassLynx software). The $(R)-$ and $(S)$-ibuprofen peaks were identifiable, discrete, and reproducible, with an accuracy (\%Deviation) of $\pm 20 \%$ and precision $(\% \mathrm{RSD}) \leq 20 \%$. To determine LOD, triplicate samples of $1 \mathrm{ng} / \mathrm{mL}(R)$ - and $(S)$-ibuprofen were prepared in human plasma and analyzed. The mean response was determined from the samples. The LOD was determined as the lowest concentration of $(R)$ - and $(S)$ ibuprofen in the samples where the mean response of the $(R)$ - and $(S)$-ibuprofen peaks was at least 3-times the response compared to the blank sample.

\subsubsection{Selectivity}

Selectivity was determined in pooled human plasma samples made from three separate lots spiked at the LOQ level. Triplicate samples were prepared. Blank human plasma samples without $(R)$ - and $(S)$-ibuprofen and $(R)$ - 
and $(S)$-flurbiprofen were also prepared using the pooled human plasma samples made from the three lots. The blank samples were visually compared to the LOQ samples for any significant interference at the retention times of $(R)$ - and $(S)$-ibuprofen and $(R)$ - and $(S)$-flurbiprofen. The acceptance criteria for selectivity were that the mean response (i.e., $\mathrm{S} / \mathrm{N})$ of $(R)$ - and $(S)$-ibuprofen in the LOQ samples were at least 5 -times the response compared to the blank samples, and there was no significant matrix interference at the retention times of $(R)$ - and $(S)$-ibuprofen and $(R)$ - and $(S)$-flurbiprofen when the blank samples were compared with the LOQ samples.

\subsubsection{Absolute Recovery}

Samples of QC-Low, QC-Mid, and QC-High were prepared in human plasma and analyzed. The $(R)$ - and $(S)$ ibuprofen peak area counts of the extracted samples were compared to the $(R)$ - and $(S)$-ibuprofen peak area counts of directly injected standards of the same concentration. Six determinations per concentration were performed and absolute recovery was calculated according to the following formula: \%Absolute recovery $=($ extracted $(R)-$ and $(S)$-ibuprofen peak area counts/unextracted $(R)$ - and $(S)$-ibuprofen peak area counts $) \times 100$.

\subsubsection{Matrix Effect}

Matrix effect, which may cause ionization suppression or enhancement of the analytes, was determined in three blank human plasma lots. Samples of QC-Low, QC-Mid, and QC-High were prepared in human plasma and in water and analyzed. The $(R)$ - and $(S)$-ibuprofen peak area counts of the plasma samples were compared to that obtained in samples prepared in water. Samples were analyzed in triplicates and the matrix effect was calculated according to the following formula: \%Matrix Effect = peak area counts in plasma-peak area counts in water/ peak area counts in water $\times 100$. Matrix effect was considered negligible if no more than $10 \%$ difference in the peak area counts of $(R)$ - and $(S)$-ibuprofen was observed in the human plasma samples compared to the samples prepared in water. Negative \%matrix effect represented ionization suppression, and positive \%matrix effect represented ionization enhancement.

\subsubsection{Dilution-Integrity}

Six aliquots of QC-High $(2500 \mathrm{ng} / \mathrm{mL})$ samples were diluted 10-fold with blank human plasma and analyzed. The acceptance criteria for $(R)$ - and $(S)$-ibuprofen were the accuracy ( $\%$ Deviation) $\pm 15 \%$ from the actual value $(250 \mathrm{ng} / \mathrm{mL})$, and precision $(\% \mathrm{RSD}) \leq 15 \%$ from the six determinations.

\subsubsection{Evaluation of Carry-over}

An aliquot of a QC-Mid sample was prepared and three injections were made, immediately followed by three injections of a blank human plasma sample. Carry-over was expressed as the percentage difference between the mean $(R)$ - and $(S)$-ibuprofen or $(R)$ - and $(S)$-flurbiprofen peak area counts in blank human plasma samples and the mean $(R)$ - and $(S)$-ibuprofen or $(R)$ - and $(S)$-flurbiprofen peak area count in the QC-Mid sample. Carry-over was considered negligible if no more than $5 \%$ of $(R)$ - and $(S)$-ibuprofen or $(R)$ - and $(S)$-flurbiprofen was observed in the blank plasma. The percentage carry-over was calculated as follows: \%Carry-over $=(B L / Q C-M i d) \times 100$, where $\mathrm{BL}=$ Mean peak area count in the blank samples (at retention times of $(R)$ - and $(S)$-ibuprofen or $(R)$ - and $(S)$-flurbiprofen), QC-Mid = Mean $(R)$ - and $(S)$-ibuprofen or $(R)$ - and $(S)$-flurbiprofen peak area count in the QC-Mid sample.

\section{Results and Discussion}

The objective of this study was to develop and validate a sensitive and enantioselective UPLC-MS/MS method for the quantitation of the $(R)$ - and $(S)$-ibuprofen in human plasma. A method was needed, which had a simple sample preparation step including derivatization, so that the resulting $(R)$ - and $(S)$-ibuprofen diastereomers could be analyzed with conventional reverse-phase chromatographic conditions.

\subsection{Method Development and Optimization}

The present analytical procedure is based upon a previously reported assay [29] with modifications to the method of detection, chromatography and sample preparation as described below.

\subsubsection{Mass Spectrometry}

The spectrofluorometric detection [29] was replaced with mass spectrometric detection. Mass spectrometry is a much improved detection technique, because it allows for the selective monitoring of the MRM transitions of $(R)$ - and $(S)$-ibuprofen and $(R)$ - and $(S)$-flurbiprofen, therefore, eliminating any interferences with co-eluting endogenous components from human plasma. In order to monitor $(R)$ - and $(S)$-ibuprofen and $(R)$ - and $(S)$-flurbiprofen using a non-chiral chromatographic system, the compounds were derivatized as described above to yield the corresponding diastereomeric amides. The amide derivatives contained a nitrogen atom, which could be ionized efficiently using ES + mode. The molecular ions were determined by the direct injection of about 100 $\mu \mathrm{g} / \mathrm{mL}$ solutions of the analytes in water/methanol: $50 / 50$ into the mobile phase flow of the same composition in the absence of a chromatographic column. The molecular ions observed for $(R)$ - and $(S)$-ibuprofen were $m / z$ 360.2, and $m / z 398.3$ for $(R)$ - and $(S)$-flurbiprofen. The cone voltage value was optimized with the most intense signal 
obtained at a cone voltage of $30 \mathrm{~V}$ for each analyte. Source temperature, desolvation temperature, and desolvation gas flow values were optimized for the highest signal and are reported in Subsection 2.2. The product ions of the analytes were determined and representative product ion mass spectra obtained in ES+ for $(R)$ - and $(S)$-ibuprofen, and for $(R)$ - and $(S)$-flurbiprofen are presented in Figure 1. The CE values were optimized to obtain the most abundant signals for the product ions. The proposed fragmentation pattern of the diastereomeric amide derivatives of $(R)$ - and $(S)$-ibuprofen, and $(R)$ - and $(S)$-flurbiprofen obtained in ES+ is presented in Figure 2. MRM transitions were created and $(R)$ - and $(S)$-ibuprofen was detected at $m / z 360.2 \rightarrow 232.1$ (CE 10 $\mathrm{eV})$, and $(R)$-and $(S)$-flurbiprofen at $m / z 398.3 \rightarrow 270.1$ $(\mathrm{CE} 10 \mathrm{eV})$. The dwell time was set to $25 \mathrm{~ms}$ for $(R)$-and $(S)$-ibuprofen as this provided sufficient sampling points during the chromatographic peaks to achieve reliable integration and, therefore, reproducible results. The dwell time for $(R)$-and $(S)$-flurbiprofen was set to $50 \mathrm{~ms}$.

\subsubsection{Liquid Chromatography}

The HPLC system using a conventional C18 column was replaced with a UPLC system using a narrow-bore Acquity UPLC ethylene bridged hybrid (BEH) phenyl column, and the mobile phase system containing a mixture of phosphate buffer and acetonitrile was replaced with a mobile phase system containing a mixture of water, ammonium acetate, formic acid, methanol and acetonitrile.

For our initial studies, an Acquity UPLC BEH C18 1.7 $\mu \mathrm{m}, 2.1 \times 100 \mathrm{~mm}$ column was tested for the separation of $(R)$ - and $(S)$-ibuprofen, and $(R)$ - and $(S)$-flurbiprofen. The mobile phase composition was solvent a: water with $0.1 \%$ formic acid and solvent b: methanol with $0.1 \%$ formic acid with the flow rate set at $0.2 \mathrm{~mL} / \mathrm{min}$. A series of isocratic chromatographic experiments were performed which are summarized as follows. Ammonium acetate $(10 \mathrm{mM})$ was added to the mobile phase, different mobile phase compositions were tested, methanol was replaced with acetonitrile in solvent $b$, various solvent $b$ compositions were prepared with different proportions of methanol and acetonitrile mixed in solvent $b$. While it was found that methanol was a selective solvent for the separation of $(R)$ - and $(S)$-ibuprofen and acetonitrile for $(R)$ - and $(S)$-flurbiprofen, no baseline separation could be achieved for these compounds with any additional changes made to the chromatographic conditions when the C18 chromatographic column was used.

Based on these findings, the $\mathrm{C} 18$ column was replaced with a Waters Acquity UPLC BEH Phenyl $1.7 \mu \mathrm{m} 2.1 \times$ $150 \mathrm{~mm}$ column. With the phenyl column, when solvent a was water with $10 \mathrm{mM}$ ammonium acetate and $0.1 \%$ formic acid, and solvent $\mathrm{b}$ was a mixture of acetonitrile/ methanol: 64/36 containing $10 \mathrm{mM}$ ammonium acetate and $0.1 \%$ formic acid, and using an isocratic run with a



(a)

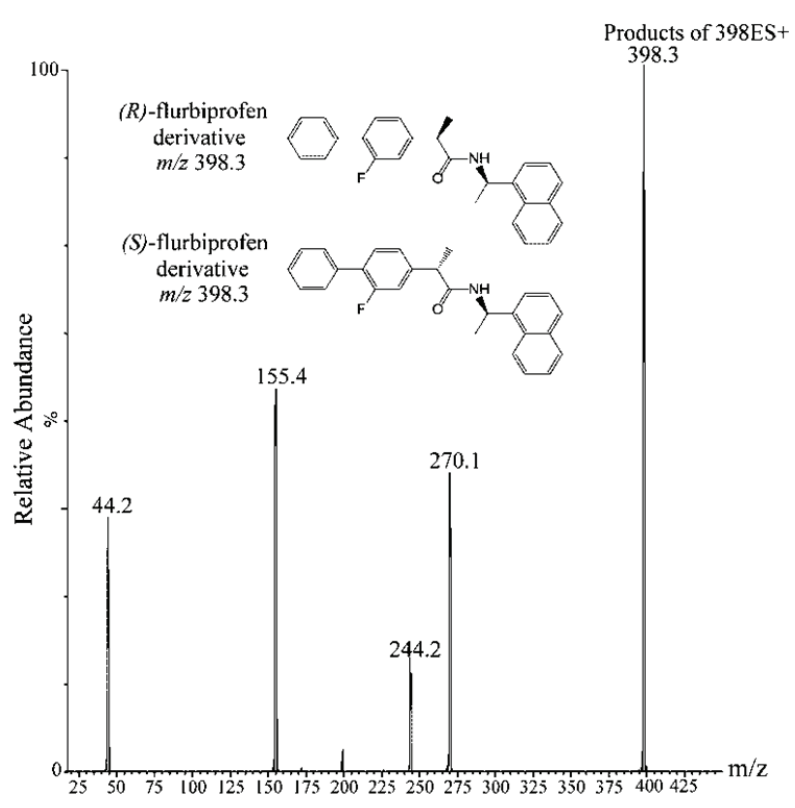

(b)

Figure 1. Representative product ion mass spectra of the diastereomeric amide derivatives of (a) (R)- and (S)-ibuprofen, and (b) $(R)$ - and $(S)$-flurbiprofen obtained in ES+ with a collision energy $(\mathrm{CE}) 10 \mathrm{eV}$.

flow rate of $0.2 \mathrm{~mL} / \mathrm{min}$, and a mobile phase composition of solvent a/solvent b: 35/65, baseline separation was achieved for $(R)$ - and $(S)$-ibuprofen, and $(R)$ - and $(S)$ flurbiprofen. The final gradient programming is detailed in Subsection 2.2. With this chromatographic column, mobile phase composition and gradient programming, $(R)$ - and $(S)$-flurbiprofen eluted at a retention time of 8.82 
R1


(1), (2) $m / z 232$

$m / z 155$

(3), (4) $m / z 270$

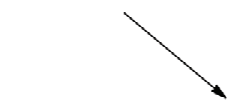

(1), (2) $m / z 206$

(3), (4) $m / z 244$
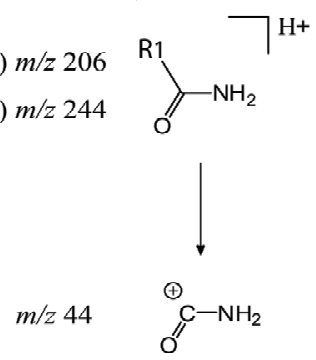

Figure 2. Proposed fragmentation pattern of diastereomeric amide derivatives of (1) (R)- and (2) (S)-ibuprofen, (3) (R)and (4) (S)-flurbiprofen obtained in ES+.

min and $10.03 \mathrm{~min}$, respectively, and $(R)$ - and $(S)$-ibuprofen eluted at a retention time $10.12 \mathrm{~min}$ and 11.07 min, respectively. A representative chromatogram of the plasma sample taken from a human volunteer $120 \mathrm{~min}$ after the oral administration of a $400 \mathrm{mg}$ ibuprofen capsule is presented in Figure 3.

\subsubsection{Sample Preparation}

The sample volume required for analysis was reduced from $0.5 \mathrm{~mL}$ [29] to $100 \mu \mathrm{L}$, and the sample preparation was simplified. The buffering step with sodium phosphate buffer was removed and the extraction solvent diethylether was replaced with methyl-tert-butyl ether which is less flammable, less toxic and easier to use. The mixing time for sample preparation was reduced, and the transfer of the organic layer was made quicker and more efficient by freezing the bottom aqueous layer in the tubes and decanting the top organic layer into a clean set of tubes. After derivatization, the step of passing the samples through solid-phase extraction silica cartridges was eliminated.

\subsection{Method Validation}

\subsubsection{Accuracy and Precision}

The results for accuracy and precision are presented in Table 1. Accuracy was expressed as the \%Deviation for the QC-Low, QC-Mid and QC-High samples. Intra-day accuracy for $(R)$-ibuprofen ranged between $-11.8 \%$ and $11.2 \%$, and for $(S)$-ibuprofen between $-8.6 \%$ and $-0.3 \%$. Inter-day accuracy for $(R)$-ibuprofen ranged between $-10.4 \%$ and $4.5 \%$, and for $(S)$-ibuprofen between $-4.8 \%$ and $-0.6 \%$. Precision was expressed as the \%RSD for the QC-Low, QC-Mid and QC-High samples. Intra-day precision for $(R)$-ibuprofen ranged between $2.2 \%$ and $10.3 \%$, and for $(S)$-ibuprofen between $1.7 \%$ and $7.0 \%$. Inter-day precision for $(R)$-ibuprofen ranged between $5.9 \%$ and $11.2 \%$, and for $(S)$-ibuprofen between $4.0 \%$ and $4.8 \%$. The method met the acceptance criteria for accuracy of $\%$ Deviation $\pm 20 \%$ for the QC-Low samples, and $\pm 15 \%$ for the QC-Mid, and QC-High samples, and for precision of $\%$ RSD $\leq 20 \%$ for QC-Low samples, and $\leq 15 \%$ for the QC-Mid, and QC-High samples. This indicated that the method was accurate and precise over the range of the assay.

\subsubsection{Linearity and Range}

Linearity of the calibration curve was evaluated in seven batches over the course of the validation. The coefficient of determination (mean $\pm \mathrm{SD}, \mathrm{n}=7$ ) for $(R$ )-ibuprofen was $\mathrm{r}^{2}=0.995 \pm 0.004$, and for $(S)$-ibuprofen $\mathrm{r}^{2}=0.996$ \pm 0.004 . The accuracy (\%Deviation) of the calibration curve levels for $(R)$-ibuprofen ranged between $-12.9 \%$ and $5.5 \%$, and for $(S)$-ibuprofen between $-4.6 \%$ and $3.4 \%$. The precision ( $\% \mathrm{RSD}$ ) of the calibration curve levels for $(R)$-ibuprofen ranged between $2.3 \%$ and $4.4 \%$, and for $(S)$-ibuprofen between $1.2 \%$ and $10.2 \%$. The calibration curve met the acceptance criterion for linearity of $r^{2} \geq 0.980$ after weighting with $1 / X^{2}$. The range of the method was established as $50 \mathrm{ng} / \mathrm{mL}$ to $5000 \mathrm{ng} / \mathrm{mL}$ where the calibration levels met the acceptance criteria of accuracy (\%Deviation) $\pm 15 \%$, and precision (\%RSD) $\leq 15 \%$. The results indicated that the calibration curve was linear, accurate, and precise over the range of the method. 
Table 1. Accuracy (intra- and inter-day) and precision (intra-and inter-day) for $(R)$ - and $(S)$-ibuprofen in human plasma.

( $R$ )-ibuprofen
$(S)$-ibuprofen

\begin{tabular}{|c|c|c|c|c|c|c|c|c|c|}
\hline \multirow{2}{*}{$\begin{array}{l}\text { Accuracy } \\
\text { QC-Low }(80 \mathrm{ng} / \mathrm{mL})\end{array}$} & \multicolumn{3}{|c|}{ Intra-Day } & \multirow{2}{*}{$\begin{array}{c}\text { Inter-Day } \\
\text { Day } 1-3 \\
(\mathrm{n}=17)\end{array}$} & \multirow{2}{*}{$\begin{array}{l}\text { Accuracy } \\
\text { QC-Low }(80 \mathrm{ng} / \mathrm{mL})\end{array}$} & \multicolumn{3}{|c|}{ Intra-Day } & \multirow{2}{*}{$\begin{array}{c}\text { Inter-Day } \\
\text { Day } 1-3 \\
(\mathrm{n}=17)\end{array}$} \\
\hline & $\begin{array}{l}\text { Day 1 } \\
(n=5)\end{array}$ & $\begin{array}{c}\text { Day } 2 \\
(n=6)\end{array}$ & $\begin{array}{c}\text { Day } 3 \\
(n=6)\end{array}$ & & & $\begin{array}{l}\text { Day 1 } \\
(\mathrm{n}=5)\end{array}$ & $\begin{array}{c}\text { Day } 2 \\
(n=6)\end{array}$ & $\begin{array}{c}\text { Day } 3 \\
(n=6)\end{array}$ & \\
\hline Mean (ng/mL) & 76.7 & 82.2 & 81.1 & 80.2 & Mean (ng/mL) & 79.7 & 79.1 & 79.6 & 79.5 \\
\hline $\mathrm{SD}(\mathrm{ng} / \mathrm{mL})$ & 2.38 & 5.11 & 3.63 & 4.38 & $\mathrm{SD}(\mathrm{ng} / \mathrm{mL})$ & 4.99 & 5.21 & 3.69 & 4.36 \\
\hline$\%$ Deviation & -4.1 & 2.7 & 1.3 & 0.2 & $\%$ Deviation & -0.3 & -1.1 & -0.5 & -0.6 \\
\hline QC-Mid (250 ng/mL) & & & & & QC-Mid (250 ng/mL) & & & & \\
\hline Mean (ng/mL) & 242 & 261 & 278 & 261 & Mean (ng/mL) & 244 & 244 & 249 & 246 \\
\hline $\mathrm{SD}(\mathrm{ng} / \mathrm{mL})$ & 22.6 & 10.8 & 14.5 & 18.9 & $\mathrm{SD}(\mathrm{ng} / \mathrm{mL})$ & 6.72 & 7.82 & 8.47 & 7.62 \\
\hline$\%$ Deviation & -3.4 & 4.4 & 11.2 & 4.5 & $\%$ Deviation & -2.3 & -2.4 & -0.5 & -1.7 \\
\hline QC-High (2500 ng/mL) & & & & & QC-High (2500 ng/mL) & & & & \\
\hline Mean (ng/mL) & 2205 & 2246 & 2267 & 2241 & Mean (ng/mL) & 2428 & 2285 & 2433 & 2379 \\
\hline $\mathrm{SD}(\mathrm{ng} / \mathrm{mL})$ & 18.1 & 71.0 & 43.9 & 54.0 & $\mathrm{SD}(\mathrm{ng} / \mathrm{mL})$ & 37.7 & 98.4 & 85.7 & 104 \\
\hline$\%$ Deviation & -11.8 & -10.2 & -9.3 & -10.4 & $\%$ Deviation & -2.9 & -8.6 & -2.7 & -4.8 \\
\hline \multicolumn{6}{|c|}{$(R)$-ibuprofen } & \multicolumn{4}{|c|}{$(S)$-ibuprofen } \\
\hline Precision & \multicolumn{3}{|c|}{ Intra-Day } & Inter-Day & Precision & \multicolumn{3}{|c|}{ Intra-Day } & Inter-Day \\
\hline QC-Low (80 ng/mL) & $\begin{array}{l}\text { Day 1 } \\
(\mathrm{n}=6)\end{array}$ & $\begin{array}{l}\text { Day } 2 \\
(n=5)\end{array}$ & $\begin{array}{c}\text { Day } 3 \\
(n=6)\end{array}$ & $\begin{array}{l}\text { Day } 1-3 \\
(\mathrm{n}=17)\end{array}$ & QC-Low (80 ng/mL) & $\begin{array}{c}\text { Day } 1 \\
(\mathrm{n}=6)\end{array}$ & $\begin{array}{l}\text { Day } 2 \\
(\mathrm{n}=5)\end{array}$ & $\begin{array}{l}\text { Day } 3 \\
(\mathrm{n}=6)\end{array}$ & $\begin{array}{l}\text { Day 1-3 } \\
(\mathrm{n}=17)\end{array}$ \\
\hline Mean (ng/mL) & 78.6 & 89.6 & 96.7 & 88.2 & Mean (ng/mL) & 89.5 & 90.0 & 89.0 & 89.4 \\
\hline $\mathrm{SD}(\mathrm{ng} / \mathrm{mL})$ & 4.88 & 8.29 & 5.73 & 9.87 & $\mathrm{SD}(\mathrm{ng} / \mathrm{mL})$ & 3.40 & 6.28 & 2.36 & 3.92 \\
\hline$\%$ RSD & 6.2 & 9.2 & 5.9 & 11.2 & $\%$ RSD & 3.8 & 7.0 & 2.6 & 4.4 \\
\hline QC-Mid (250 ng/mL) & & & & & QC-Mid (250 ng/mL) & & & & \\
\hline Mean (ng/mL) & 268 & 298 & 274 & 279 & Mean (ng/mL) & 271 & 267 & 252 & 263 \\
\hline $\mathrm{SD}(\mathrm{ng} / \mathrm{mL})$ & 9.25 & 12.5 & 10.3 & 16.4 & $\mathrm{SD}(\mathrm{ng} / \mathrm{mL})$ & 4.48 & 5.20 & 8.58 & 10.5 \\
\hline$\%$ RSD & 3.4 & 4.2 & 3.7 & 5.9 & $\%$ RSD & 1.7 & 1.9 & 3.4 & 4.0 \\
\hline QC-High (2500 ng/mL) & & & & & QC-High $(2500 \mathrm{ng} / \mathrm{mL})$ & & & & \\
\hline Mean $(n g / m L)$ & 1912 & 2121 & 2040 & 2018 & Mean (ng/mL) & 2219 & 2121 & 2074 & 2139 \\
\hline $\mathrm{SD}(\mathrm{ng} / \mathrm{mL})$ & 81.7 & 218 & 44.5 & 149 & $\mathrm{SD}(\mathrm{ng} / \mathrm{mL})$ & 79.6 & 110 & 68.4 & 103 \\
\hline$\%$ RSD & 4.3 & 10.3 & 2.2 & 7.4 & $\%$ RSD & 3.6 & 5.2 & 3.3 & 4.8 \\
\hline
\end{tabular}

$\mathrm{SD}=$ Standard Deviation; \%Deviation $=[($ measured amount/added amount $) \times 100]-100[\%] ; \%$ RSD $=$ Relative Standard Deviation $[\%]$.

\subsubsection{Limit of Quantitation, Selectivity, Limit of Detection}

LOQ was determined in six replicates of the $50 \mathrm{ng} / \mathrm{mL}$ calibration standard. The mean response of the $(R)$ - and $(S)$-ibuprofen peaks in the samples was higher than 5 -times the response obtained in the blank sample. The accuracy (\%Deviation) for $(R)$-ibuprofen ranged between $-7.1 \%$ and $7.3 \%$ and for $(S)$-ibuprofen between $-0.4 \%$ and $5.2 \%$. The precision ( $\% \mathrm{RSD})$ for $(R)$-ibuprofen was $5.8 \%$ and for $(S)$-ibuprofen $2.0 \%$. Results for the determination of LOQ met the acceptance criteria of the mean response which was at least 5-times the response compared to the blank sample, accuracy (\%Deviation) $\pm 20 \%$ and precision $\%$ RSD $\leq 20 \%$, and the $(R)$ - and $(S)$-ibuprofen peaks were identifiable, discrete, and reproducible. The method was accurate and precise with an established LOQ of $50 \mathrm{ng} / \mathrm{mL}$ of $(R)$ - and $(S)$-ibuprofen requiring $100 \mu \mathrm{L}$ of sample. The selectivity of the method was investigated in triplicate samples of pooled human plasma made from three separate lots spiked at LOQ level. Blank human plasma samples from three separate lots were also prepared. The selectivity met the acceptance criteria that the mean response for $(R)$ - and $(S)$ ibuprofen was at least 5-times the response compared to the blank samples, and there was no significant interference at the retention times of $(R)$ - and $(S)$-ibuprofen and 
$(R)$ - and $(S)$-flurbiprofen when the blank human plasma samples were compared to the LOQ samples. The results indicated that the method was selective for these analytes. LOD was determined in triplicate samples of $1 \mathrm{ng} / \mathrm{mL}$ $(R)$ - and $(S)$-ibuprofen prepared in human plasma. The mean response (i.e., $\mathrm{S} / \mathrm{N})$ of the $(R)$ - and $(S)$-ibuprofen peaks in the samples was higher than 3-times the response obtained in the blank sample. The method had an established LOD of $1 \mathrm{ng} / \mathrm{mL}$ of $(R)$ - and $(S)$-ibuprofen requiring $100 \mu \mathrm{L}$ of sample. Representative chromatograms of LOD (1 ng/mL), and blank human plasma samples are presented in Figure 4.

\subsubsection{Absolute Recovery and Matrix Effect}

The mean \%Absolute recovery values $(n=6)$ for $(R)$-ibuprofen for QC-Low, QC-Mid and QC-High were $81.9 \%, 86.9 \%$ and $84.5 \%$, respectively, and for $(S)$-ibuprofen $82.2 \%, 83.4 \%$ and $83.7 \%$, respectively. The results indicated that $(R)$ - and $(S)$-ibuprofen were extracted relatively uniformly over the concentration range of the assay. The mean \%Matrix effect values $(\mathrm{n}=9)$ for $(R)$-ibuprofen for QC-Low, QC-Mid, and QC-High were



Figure 3. A representative chromatogram of the plasma sample taken from a human volunteer 120 min after the oral administration of a $400 \mathrm{mg}$ ibuprofen capsule. The MRM signal of $m / z, 360.2 \rightarrow 232.1$ for $(R)$ - and $(S)$-ibuprofen is on the top, and $m / z 398.3 \rightarrow 270.1$ for internal standards $(R)$ - and $(S)$-flurbiprofen is on the bottom (the measured $(R)$ - and $(S)$-ibuprofen concentrations were $803 \mathrm{ng} / \mathrm{mL}$ and $1087 \mathrm{ng} / \mathrm{mL}$, respectively).
$1.3 \%, 2.2 \%$ and $-2.5 \%$, respectively, and for $(S)$ - ibuprofen $2.1 \%,-6.2 \%$, and $-2.0 \%$, respectively. The results met the acceptance criterion of \%Matrix effect of no more than $10 \%$. This indicated that the matrix effect from human plasma contributing to the ionization suppression or enhancement of $(R)$ - and $(S)$-ibuprofen was negligible.

\subsubsection{Dilution Integrity and Evaluation of Carry-over} After the 10-fold dilution of the six aliquot of the QCHigh samples, the accuracy $(\%$ Deviation, $\mathrm{n}=6)$ for $(R)$-ibuprofen ranged between $-5.5 \%$ and $4.1 \%$, and for $(S)$-ibuprofen between $-7.9 \%$ and $5.6 \%$. The precision $(\% \mathrm{RSD}, \mathrm{n}=6)$ for $(R)$-ibuprofen was $3.7 \%$ and for $(S)$-ibuprofen $4.5 \%$. The results met the acceptance criteria of accuracy ( $\%$ Deviation) $\pm 15 \%$ from the actual value $(250 \mathrm{ng} / \mathrm{mL})$, and precision $(\% \mathrm{RSD}) \leq 15 \%$. This indicated that samples exceeding the calibration curve concentrations could be diluted 10-fold to bring them into the range of the assay with accuracy and precision. Carry-over between injections of the QC-Mid and blank plasma samples for $(R)$-ibuprofen was $0.4 \%$, for $(S)$-ibuprofen $1.0 \%$, for $(R)$-flurbiprofen $0.1 \%$, and for $(S)$-flurbiprofen $0.1 \%$; all met the acceptance criteria of no more than $5 \%$.

\subsubsection{Pharmacokinetics of $(R)$ - and $(S)$-ibuprofen in humans}

Healthy human volunteers were involved in a pharmacokinetic study following the administration of a single oral dose of $400 \mathrm{mg}$ ibuprofen capsules. The detailed pharmacokinetic results of this study will be published elsewhere. Here we report the results of four randomly selected subjects to demonstrate the applicability of the developed and validated method to determine the $(R)$ and $(S)$-ibuprofen concentrations in human plasma. Plasma samples were collected at various time intervals up to $300 \mathrm{~min}$ following the ibuprofen dose. A representative plasma concentration $v s$. time plot obtained from one subject is presented in Figure 5. $(R)$ - and $(S)$-ibuprofen concentrations increased during the first $90 \mathrm{~min}$ reaching a peak value of $15,026 \mathrm{ng} / \mathrm{mL}$ for $(R)$-ibuprofen, and $18,915 \mathrm{ng} / \mathrm{mL}$ for $(S)$-ibuprofen. After that time, the drug concentrations decreased gradually and remained above $3100 \mathrm{ng} / \mathrm{mL}$ for $(R)$-ibuprofen, and $5800 \mathrm{ng} / \mathrm{mL}$ for $(S)$-ibuprofen at the final sampling time of $300 \mathrm{~min}$. Pharmacokinetic analysis of the plasma concentration data obtained from the four subjects was performed using a non-compartmental analysis (PK Solutions 2.0, Montrose, CO, USA). The results were normalized to body weights. The estimated pharmacokinetic parameters for $(R)$-ibuprofen were as follows (mean $\pm \mathrm{SD}, \mathrm{n}=$ 4): terminal elimination half-life $\left(\mathrm{t}_{1 / 2}\right): 83.8 \pm 8.62 \mathrm{~min}$, $\mathrm{T}_{\max }: 37.5 \pm 8.66 \mathrm{~min}, \mathrm{C}_{\max }: 14,076 \pm 3160 \mathrm{ng} / \mathrm{mL}$, apparent volume of distribution $(\mathrm{V} / \mathrm{F}): 350 \pm 50.4 \mathrm{~mL} / \mathrm{kg}$, apparent oral clearance (CL/F): $2.91 \pm 0.519 \mathrm{~mL} / \mathrm{min} / \mathrm{kg}$, 

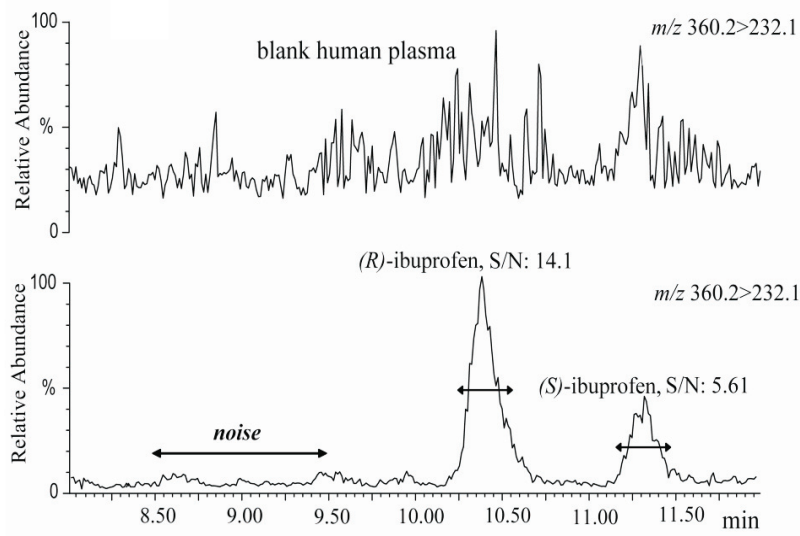

(a)


(b)

Figure 4. Limit of detection (LOD, $1 \mathrm{ng} / \mathrm{mL}$ ) and blank human plasma samples. (a) shows the MRM $\mathrm{m} / z, 360.2 \rightarrow$ 232.1 for $(R)$ - and $(S)$-ibuprofen in blank human plasma (top), and in the LOD sample (bottom) (the signal-to-noise $[\mathrm{S} / \mathrm{N}]$ values are reported on the top of the $(R)$ - and (S)-ibuprofen peaks). (b) shows the MRM $\mathrm{m} / \mathrm{z} 398.3 \rightarrow$ 270.1 for $(R)$ - and $(S)$-flurbiprofen, internal standard, in blank human plasma (top), and in the LOD sample (bottom).

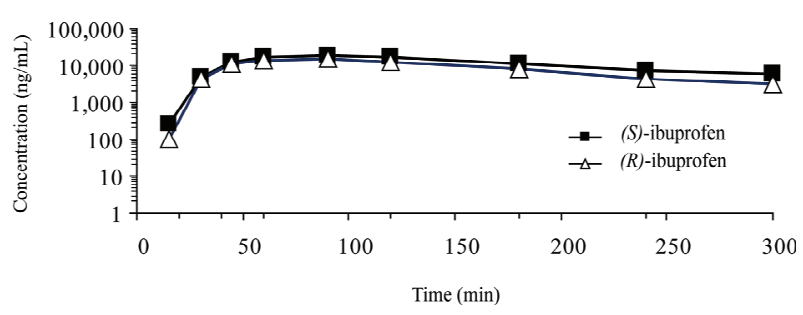

Figure 5. Concentration of $(R)$ - and $(S)$-ibuprofen vs. time profile obtained from a volunteer following a single oral dose of a $400 \mathrm{mg}$ ibuprofen capsule.

and mean residence time $\left(\mathrm{MRT}_{\mathrm{INF}}\right): 142 \pm 23.4 \mathrm{~min}$; and for $(S)$-ibuprofen (mean $\pm \mathrm{SD}, \mathrm{n}=4)$ : terminal elimination half-life $\left(\mathrm{t}_{1 / 2}\right): 108 \pm 14.6 \mathrm{~min}, \mathrm{~T}_{\max }: 41.3 \pm 7.50 \mathrm{~min}$,
$\mathrm{C}_{\max }: 15,779 \pm 4720 \mathrm{ng} / \mathrm{mL}$, apparent volume of distribution $(\mathrm{V} / \mathrm{F}): 325 \pm 91.1 \mathrm{~mL} / \mathrm{kg}$, apparent oral clearance $(\mathrm{CL} / \mathrm{F}): 2.06 \pm 0.303 \mathrm{~mL} / \mathrm{min} / \mathrm{kg}$, and mean residence time $\left(\mathrm{MRT}_{\mathrm{INF}}\right): 173 \pm 16.1 \mathrm{~min}$.

In summary, the results show that following the oral administration of $400 \mathrm{mg}$ racemic ibuprofen, both enantiomer showed rapid absorption and stereoselective disposition. For $(S)$-ibuprofen, the plasma concentrations peaked at $41.3 \mathrm{~min}$, reached higher levels (i.e., 15,779 $\mathrm{ng} / \mathrm{mL}$ ), had higher terminal elimination half life (108 $\mathrm{min})$, apparent volume of distribution $(325 \mathrm{~mL} / \mathrm{kg})$, mean residence time $(173 \mathrm{~min})$, and lower apparent oral clearance $(2.06 \mathrm{~mL} / \mathrm{min} / \mathrm{kg})$ than those values for $(R)$-ibuprofen (i.e., $\mathrm{T}_{\max }$ : $37.5 \mathrm{~min}, \mathrm{C}_{\max }$ : $14,076 \mathrm{ng} / \mathrm{mL}, \mathrm{t}_{1 / 2}: 83.8 \mathrm{~min}$, $\mathrm{V} / \mathrm{F}: 350 \mathrm{~mL} / \mathrm{kg}, \mathrm{MRT}_{\mathrm{INF}}: 142 \mathrm{~min}$, and CL/F: $2.91 \mathrm{~mL} /$ $\mathrm{min} / \mathrm{kg}$, respectively). These findings are consistent with the data of in vivo chiral inversion of $(R)$-ibuprofen to $(S)$-ibuprofen reported in the literature [9-11].

\section{Conclusions}

An enantioselective modified UPLC-MS/MS method was developed and validated for the quantitation of $(R)$ and $(S)$-ibuprofen enantiomers in human plasma. Compared to previously published assays, sample preparation was simplified without the need of post-derivatization sample clean-up [29], sample volume required for analysis was reduced at least 5 -fold $[6,25,29]$, the range of the calibration curve was extended into the three-digit range $[23,25,29]$, baseline separation of $(R)$ - and $(S)$-ibuprofen was achieved [20,25] and the sensitivity was increased 2-fold [23-25,29]. The assay was validated with a LOQ of $50 \mathrm{ng} / \mathrm{mL}$, however, the sensitivity of the assay proved to be significantly higher with a LOD of $1 \mathrm{ng} / \mathrm{mL}$. It was not necessary to validate the method at a LOQ less than $50 \mathrm{ng} / \mathrm{mL}$, because of the high concentration of the samples analyzed during this study. However, the LOQ of the present assay can be easily extended to the low $\mathrm{ng} / \mathrm{mL}$ levels if future studies require a methodology with significantly higher sensitivity. The assay demonstrated good reproducibility and was applied to investigate the pharmacokinetics of ibuprofen enantiomers following oral administration of the racemate drug.

\section{Acknowledgements}

The authors would like to thank Dr. Cathy Boscarino for her valuable work with the design of the human study; Mrs. Debbie Kerrigan-Brown, Ingrid Smith, Christina Powlesland, and Mr. Kevin Hofer, Robert Limmer, Doug Saunders, Jan Pope for their excellent technical support of the human study. This work was funded by a research contract with Defence Research and Development Canada; contract number W7711-088126. 


\section{References}

[1] R. D. Knihinicki, K. M. Williams and R. O. Day, "Chiral Inversion of 2-Arylpropionic Acid Non-Steroidal AntiInflammatory Drugs-I. In Vitro Studies of Ibuprofen and Flurbiprofen," Biochemical Pharmacology, Vol. 38, No. 24, December 1989, pp. 4389-4395.

[2] S. M. Sanins, W. J. Adams, D. G. Kaiser, G. W. Halstead, J. Hosley, H. Barnes and T. A. Baillie, "Mechanistic Studies on the Metabolic Chiral Inversion of R-Ibuprofen in the Rat," Drug Metabolism and Disposition, Vol. 19, No. 2, March-April 1991, pp. 405-410.

[3] S. Fournel and J. Caldwell, "Metabolic Chiral Inversion of 2-Phenylpropionic Acid in Rat, Mouse and Rabbit," Biochemical Pharmacology, Vol. 35, No. 23, December 1986, pp. 4153-4159.

[4] J. Caldwell, A. J. Hull and S. Fournel-Gigleux, "The Metabolic Chiral Inversion and Dispositional Enantioselectivity of the 2-Arylpropionic Acids and their Biological Consequences," Biochemical Pharmacology, Vol. 37, No. 1, January 1988, pp. 105-114.

[5] D. G. Kaiser, G. J. Van Giessen, R. J. Reischer and W. J. Wechter, "Isomeric Inversion of Ibuprofen (R)-Enantiomer in Humans," Journal of Pharmaceutical Sciences, Vol. 65, No. 2, February 1976, pp. 269-273.

[6] T. A. Baillie, W. J. Adams, D. G. Kaiser, L. S. Olanoff, G. W. Halstead, H. Harpootlian and G. J. Van Giessen, "Mechanistic Studies of the Metabolic Chiral Inversion of (R)-Ibuprofen in Humans," Journal of Pharmacology and Experimental Therapeutics, Vol. 249, No. 2, May 1989, pp. 517-523.

[7] S. S. Adams, P. Bresloff and C. G. Mason, "Pharmacological Differences Between Optical Isomers of Ibuprofen: Evidence for Metabolic Inversion of the (-)-Isomer," Journal of Pharmacy and Pharmacology, Vol. 28, No. 3, March 1976, pp. 256-257.

[8] A. J. Hutt and J. Caldwell, "The Metabolic Chiral Inversion of 2-Aryl Propionic Acids-A Novel Route with Pharmacological Consequences," Journal of Pharmacy and Pharmacology, Vol. 35, No. 11, November 1983, pp. 693-704.

[9] A. J. Hutt and J. Caldwell, "The Importance of Stereochemistry in the Clinical Pharmacokinetics of the 2Arylpropionic Acid Non-Steroidal Anti-Inflammatory Drugs," Clinical Pharmacokinetics, Vol. 9, No. 4, JulyAugust 1984, pp. 371-373.

[10] S. C. Tan, B. K. Patel, S. H. D. Jackson, C. G. Swift, and J. Hutt, "Stereoselectivity of Ibuprofen Metabolism and Pharmacokinetics Following the Administration of the Racemate to Healthy Volunteers," Xenobiotica, Vol. 32, No. 8, August 2002, pp. 683-697.

[11] H. Hao, G. Wang and J. Sun, "Enantioselective Pharmacokinetics of Ibuprofen and Involved Mechanisms," Drug Metabolism Reviews, Vol. 73, No. 1, January 2005, pp. 215-234.

[12] E. J. Lee, K. Williams, R. Day, G. Graham and D. Champion, "Stereoselective Disposition of Ibuprofen Enantiomers in Man," British Journal of Clinical Pharmacology,
Vol. 19, No. 5, May 1985, pp. 669-674.

[13] S. C. Tan, B. K. Patel, S. H. Jackson, C. G. Swift and A. J. Hutt, "Influence of Age on the Enantiomeric Disposition of Ibuprofen in Healthy Volunteers," British Journal of Clinical Pharmacology, Vol. 55, No. 6, June 2003, pp. 579-587.

[14] G. F. Lockwood, K. S. Albert, G. J. Szpunar and J. G. Wagner, "Pharmacokinetics of Ibuprofen in Man-III: Plasma Protein Binding," Journal of Pharmacokinetics \& Biopharmaceutics, Vol. 11, No. 5, October 1983, pp. 469-482.

[15] J. K. Paliwal, D. E Smith, S. R Cox, R. R Berardi, V. A. Dunn-Kucharski and G. H. Elta, "Stereoselective, Competitive, and Nonlinear Plasma Protein Binding of Ibuprofen Enantiomers as Determined in Vivo in Healthy Subjects," Journal of Pharmacokinetics and Biopharmaceutics, Vol. 21, No. 2, April 1993, pp. 145-161.

[16] J. C. Nielsen, P. Bjerring, L. Arendt-Nielsen and K.-J. Petterson, "A Double-Blind, Placebo Controlled, Crossover Comparison of the Analgesic Effect of Ibuprofen $400 \mathrm{Mg}$ and $800 \mathrm{Mg}$ on Laser-Induced Pain," British Journal of Clinical Pharmacology, Vol. 30, No. 5, November 1990, pp. 711-715.

[17] P. Camilleri and C. Dyke, "Effect of ${ }^{2} \mathrm{H}_{2} \mathrm{O}$ on the Resolution of the Optical Isomers of Ibuprofen on an $\mathrm{A}_{1}$-Acid Glycoprotein Column," Journal of Chromatography A, Vol. 518, June 1990, pp. 277-281.

[18] S. Menzel-Soglowek, G. Geisslinger and K. Brune, "Stereoselective High-Performance Liquid Chromatographic Determination of Ketoprofen, Ibuprofen and Fenoprofen in Plasma Using a Chiral Alpha 1-Acid Glycoprotein Column," Journal of Chromatography A, Vol. 532, No. 2, November 1990, pp. 295-303.

[19] K. J. Pettersson and A. Olsson, "Liquid Chromatographic Determination of The Enantiomers of Ibuprofen in Plasma Using a Chiral AGP Column," Journal of Chromatography A, Vol. 563, No. 2, February 1991, pp. 414-418.

[20] W. Naidong and J. W. Lee, "Development and Validation of a Liquid Chromatographic Method for the Quantitation of Ibuprofen Enantiomers in Human Plasma," Journal of Pharmaceutical and Biomedical Analysis, Vol. 12, No. 4, April 1994, pp. 551-556.

[21] H. Y. Aboul-Enein, A. Van Overbeke, G. Vander Weken, W. Baeyens, H. Oda, P. Deprez and A. De Kruif, "HPLC on Chiralcel OJ-R for Enantiomer Separation and Analysis of Ketoprofen, from Horse Plasma, as the 9-Aminophenanthrene Derivative," Journal of Pharmacy and Pharmacology, Vol. 50, No. 3, March 1998, pp. 291-296.

[22] K. Tachibana and A. Ohnishi, "Reversed-Phase Liquid Chromatographic Separation of Enantiomers on Polysaccharide Type Chiral Stationary Phases," Journal of Chromatography A, Vol. 906, No. 1-2, January 2001, pp. 127-154.

[23] X. W. Teng, S.W. Wang and N. M. Davies, "Stereospecific High-Performance Liquid Chromatographic Analysis of Ibuprofen in Rat Serum," Journal of Chromatography $B$, Vol. 796, No. 2, November 2003, pp. 225-231. 
[24] A. Reddy, M. Hashim, Z. Wang. L. Penn, C. J. Stankovic, D. Burdette, N. Surendran and H. Cai, "A Novel Method for Assessing Inhibition of Ibuprofen Chiral Inversion and its Application in Drug Discovery," International Journal of Pharmaceutics, Vol. 335, No. 1-2, April 2007, pp. 63-69.

[25] P. S. Bonato, M. P. Del Lama and R. Carvalho, "Enantioselective Determination of Ibuprofen in Plasma by High-Performance Liquid Chromatography-Electrospray Mass Spectrometry," Journal of Chromatography B, Vol. 796, No. 2, November 2003, pp. 413-420.

[26] E. J. D. Lee, K. M. Williams, G. G. Graham, R. O. Day, and G. D. Champion, "Liquid Chromatographic Determination and Plasma Concentration Profile of Optical Isomers of Ibuprofen in Humans," Journal of Pharmaceutical Sciences, Vol. 73, No. 11, November 1984, pp. 1542-1544.

[27] R. Mehvar, F. JamaII and F. M. Pasutto, "LiquidChromatographic Assay of Ibuprofen Enantiomers in Plasma," Clinical Chemistry, Vol. 34, No. 3, March 1988, pp. 493-496.

[28] C. H. Lemko, G. Caille and R. T. Foster, "Stereospecific High-Performance Liquid Chromatographic Assay of Ibuprofen: Improved Sensitivity and Sample Processing Efficiency," Journal of Chromatography A, Vol. 619, No. 2, September 1993, pp. 330-335.

[29] S. C. Tan, S. H. D Jackson, C. G Swift and A. J. Hutt, "Enantiospecific Analysis of Ibuprofen by High Performance Liquid Chromatography: Determination of Free and Total Drug Enantiomer Concentrations in Serum and Urine," Chromatographia, Vol. 46, No. 1-2, July 1997, pp. 23-32.

[30] M. J. Hilton and K. V. Thomas, "Determination of Selected Human Pharmaceutical Compounds in Effluent and Surface Water Samples by High-Performance Liquid Chromatography-Electrospray Tandem Mass Spectrome- try," Journal of Chromatography A, Vol. 1015, No. 1-2, October 2003, pp. 129-141.

[31] R. Leverence, M. J. Avery, O. Kavetskaia, H. Bi, C. E. Hop and A. I. Gusev, "Signal Suppression/Enhancement in HPLC-ESI-MS/MS from Concomitant Medications," Biomedical Chromatography, Vol. 21, No. 11, November 2007, pp. 1143-1150.

[32] S. Y. Chang, W. Li, S. C. Traeger, B. Wang, D. Cui, H. Zhang, B. Wen and A.D. Rodrigues, "Confirmation that Cytochrome P450 2C8 (CYP2C8) Plays a Minor Role in (S)-(+)- and (R)-(-)-Ibuprofen Hydroxylation in Vitro," Drug Metabolism and Disposition, Vol. 36, No. 12, December 2008, pp. 2513-2522.

[33] G. D. Mendes, F. D. Mendes, C. C. Domingues, R. A de Oliveira, M. A. da Silva, L. S. Chen, J. O. Ilha, C. E. Fernandes and G. De Nucci, "Comparative Bioavailability of Three Ibuprofen Formulations in Healthy Human Volunteers," International Journal of Clinical Pharmacology and Therapeutics, Vol. 46, No. 6, June 2008, pp. 309318.

[34] P. Seideman, F. Lohrer, G. G. Graham, M. W. Duncan, K. M. Williams and R. O. Day, "The Stereoselective Disposition of the Enantiomers of Ibuprofen in Blood, Blister and Synovial Fluid," British Journal of Clinical Pharmacology, Vol. 38, No. 3, September 1994, pp. 221-227.

[35] B. A. Way, T. R. Wilhite, C. H. Smith and M. Landt, "Measurement of Plasma Ibuprofen by Gas Chromatography-Mass Spectrometry," Journal of Clinical Laboratory Analysis, Vol. 11, No. 6, April 1997, pp. 336-339.

[36] Á. Sebők, A. Vasanits-Zsigrai, Gy. Palkó, Gy. Záray and I. Molnár-Perl, "Identification and Quantification of Ibuprofen, Naproxen, Ketoprofen and Diclofenac Present in Waste-Waters, as their Trimethylsilyl Derivatives, by Gas Chromatography Mass Spectrometry," Talanta, Vol. 76, No. 3, July 2008, pp. 642-650. 